\section{Vol. 66, No. 25}

In the report "Multistate Outbreak of Salmonella Anatum Infections Linked to Imported Hot Peppers - United States, May-July 2016," on page 663, the footnote ( 9 ) at the bottom of the page should have read "Louisiana (two)."

\section{Vol. 66, No. 29}

In the announcement on World Hepatitis Day on page 794, the title should have read "World Hepatitis Day — July 28, 2017." 